\title{
Slip Flow Modeling trough a Rarefied Nitrogen Gas between two Coaxial Cylinders
}

\author{
Souheila Boutebba, Wahiba Kaabar \\ Department of Chemistry, Frères Mentouri University, Constantine, Algeria \\ bouteb.souha@yahoo.fr
}

\begin{abstract}
The slip flow simulation through a rarefied nitrogen gas between coaxial cylinders is studied. The flow between a heated tungsten wire and an enclosedrarefied gas $\left(N_{2}\right)$ has been studied numerically, gas temperatures range from $300 \mathrm{~K}$ in envelope wall to $2700 \mathrm{~K}$ at the filament. These temperatures generate large temperature gradients in the region of the inner cylinder where local thermodynamic equilibrium no longer applies.Temperature, density and velocity fields are calculated. This study has been carried out using the Computational Fluid Dynamics (CFD) computer code Fluent (Fluent ${ }^{\circledR}$ 6.3).

Numerical results obtained by using the CFD computer code Fluentare compared totheanalytical solution.
\end{abstract}

Keywords:Simulation, CFD, rarefied nitrogen gas, slip flow regime

\section{Introduction}

Computational fluid dynamics (CFD) is becoming more and more an engineering tool to predict flows in various types of equipment [1].

The problem of flow through rarefied gases, confined between concentric cylinders is very frequent in many technological applications, such as Pirani and diaphragm gauges for instrumentation, monitoring and control of vacuum processes or micro heat exchangers in microfluidics [2].

The slip flow regime is a slightly rarefied regime of grand importance for gas flow [3]. It corresponds to a Knudsen number between $10^{-3}$ and $10^{-1}$. The Knudsen layer has an important role in the slip flow Regime [4]. In the slip flow regime, the Navier-Stokes equations are valid in the mass flow; but the gas is not in local thermodynamic equilibrium near the wall in said layer Knudsen. In this region, different effects of rarefaction are exposed, counting the presence of a non-negligible temperature jump at the walls [5].

Rarefied gas flow simulation is possible by the commercial CFD code Fluent by using the "Low Pressure Boundary Slip" (LPBS). Pitakarnnop and al [4], used the LPBS method in slip flow regime for the simulation of triangular and trapezoidal microchannels for predicting slip velocity.

In the present study, the problem of heat transfer, in the slip flow regime, through a rarefied nitrogen gas confined between two coaxial cylinders is resolved numerically based on the Navier-Stokes and Fourier equations. The numerical simulation was performed with the fluid dynamic computer code, Fluent [6]. Fluent is a state of the art CFD (Computational Fluid Dynamics) computer package for modelling fluid flow and heat transfer problems in complex geometries. This program originates from work described by Patankar [7]. The LPBS method proposed in Fluent for implementing slip conditions is used. This work was performed by using the Navier-Stokes and Fourier equations and by an analytical solution:The numerical results obtained by using the code Fluent are compared with the results obtained by the analytical solution. The effect of the meshes type is tested. 


\section{Problem Definition and Numerical Simulation}

A long cylinder is considered with an axial tungsten wire having a diameter of $1.5 \mathrm{~mm}$ and a length of 23.5 $\mathrm{mm}$, the wire is modeled as a solid cylinder. The external cylinder is defined to be $75 \mathrm{~mm}$ in diameter and 250 $\mathrm{mm}$ in length. The filling rarefied gas is nitrogen $\left(\mathrm{N}_{2}\right)$. Boundary conditions are set equal to $2700 \mathrm{~K}$ for the filament and $300 \mathrm{~K}$ for the external cylinder. A filling pressure of $10 \mathrm{~Pa}$ is used to maintain a slip regime flow where the number of Knudsen $\mathrm{Kn}=0.02$ and therefore a conduction heat transfer inside the enclosure. The transport properties of the fluid (viscosity, thermal conductivity, and heat capacity) are defined as temperature dependent polynomials [8]-[9].

By means of the CFD code Fluent, the numerical simulation was carried out by solving the Navier-Stokes and Fourier equations.For the slip boundary conditions the Maxwell's first order model is employed with anexpression of the mean free path adjustable by the value of the Lennard-Jones length. The mean free path, $\lambda$, is calculated in this manner [6]:

$$
\lambda=\frac{k_{B} T}{\sqrt{2} \pi \sigma_{d}^{2} P}
$$

Where $\mathrm{T}$ is the temperature, $\mathrm{P}$ the pressure and $\sigma$ the Lennard-Jones characteristic length of the gas and $\mathrm{K}_{\mathrm{B}}$ is the Boltzmann constant.

The boundary condition of temperature jump employed by the Maxwell model in the Fluent code are as follows:

$$
T_{\omega}-T_{g}=2\left(\frac{2-\alpha_{T}}{\alpha_{T}}\right) K n \frac{\partial T}{\partial n} \approx 2\left(\frac{2-\alpha_{T}}{\alpha_{T}}\right) \frac{\lambda}{\delta}\left(T_{g}-T_{c}\right)
$$

Where

$$
T_{g}=\frac{T_{\omega}+\beta T_{c}}{1+\beta}
$$

and

$$
\beta=\frac{2\left(2-\alpha_{T}\right)}{\alpha_{T} \delta}
$$

$g, \mathbf{w}$ and $c$ indicate gas, wall and cell-center velocities. $\boldsymbol{\delta}$ is the distance from cell center to the wall. $\boldsymbol{\alpha}_{\mathbf{T}}$ is the thermal accommodation coefficient.

The numerical simulations are carried out by means of the finite volume method for a nitrogen gas flow. Double precision calculations are done with second order discretization scheme for a better accuracy, density and pressure fields are obtained.

\section{Analytical solution}

The temperature distribution between two cylinders in the slip regime can be calculated from the energy conservation [10]:

$$
m n c_{v} \frac{\partial T}{\partial t}=\frac{1}{r} \frac{\partial}{\partial r}(r k) \frac{\partial T}{\partial r}
$$

Where $r$ is the radial coordinate between the cylinders, $n$ is the gas number density, $k$ is the gas thermal conductivity, $t$ is the time and $c_{v}$ is the annular specific isochoric heat capacity.

In the steady state case $\partial T / \partial t=0$ and in the slip flow regime, the gas temperature next to wall can be taken equal to the wall temperature, so the Equation (5) can be solved analytically [10].

The temperature profiles $\mathrm{T}(\mathrm{r})$ will be compared in the Results section with the temperature distributions obtained by the CFD code Fluent (Navier-Stokes and Fourier equations). 


\section{Results and Discussion}

A non-uniform grid is created with 40000 quadrilateral cells (Fig.1). The mesh is refined near the limits to improve the calculations near the solid-gas interfaces, especially the filament-gas interface (kundsen layer). Simulations are carried out for the nitrogen gas flow.

The choice of the mesh type and the nodes number was decided after performing several calculations with different types of mesh and number of nodes. The definition of the geometry and the generation of the mesh were carried out using the generator code Gambit 2.3.30.

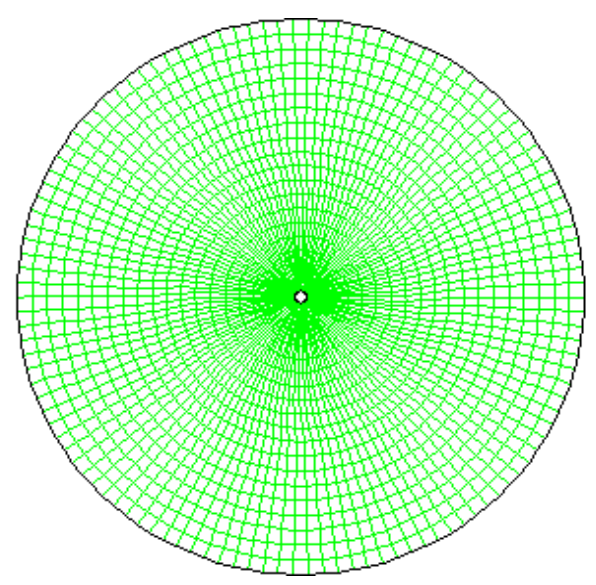

Fig.1: Geometry and mesh

The precision of the results depends on the type and fineness of mesh. The mesh influence on the solution is studied. For this purpose, the temperature distribution as a function of the radial displacement (according to the radius) is computed numerically and analytically.

Four types of mesheshave been tested. Figure 3 shows the structure of these meshes. The first mesh is normal (a), the second one is progressive (b), the third and fourth meshes are uniform with a high (c) or low(d) mesh density.

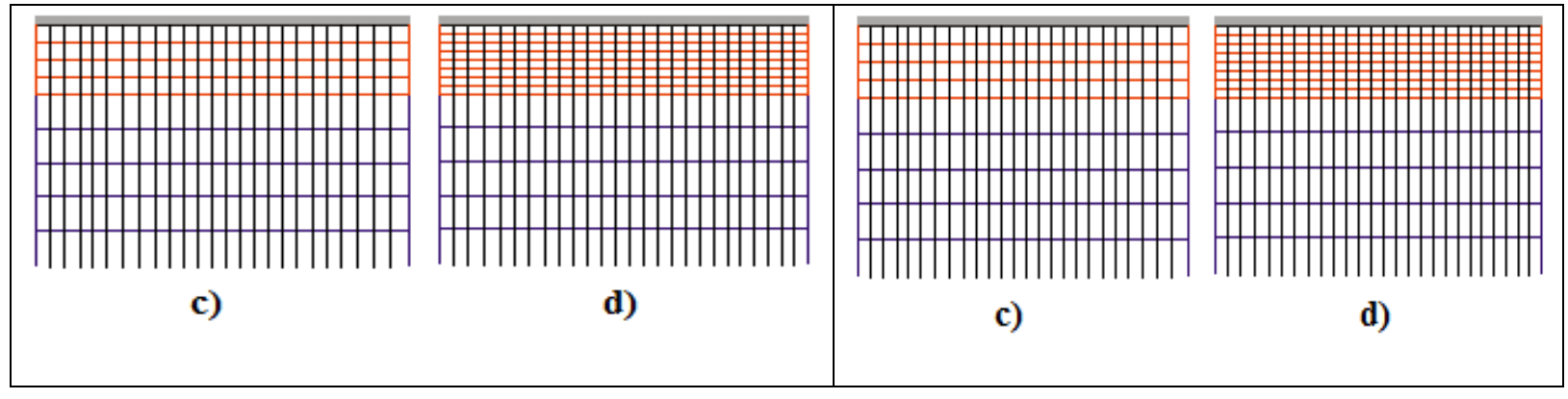

Fig.2:Types of meshes 


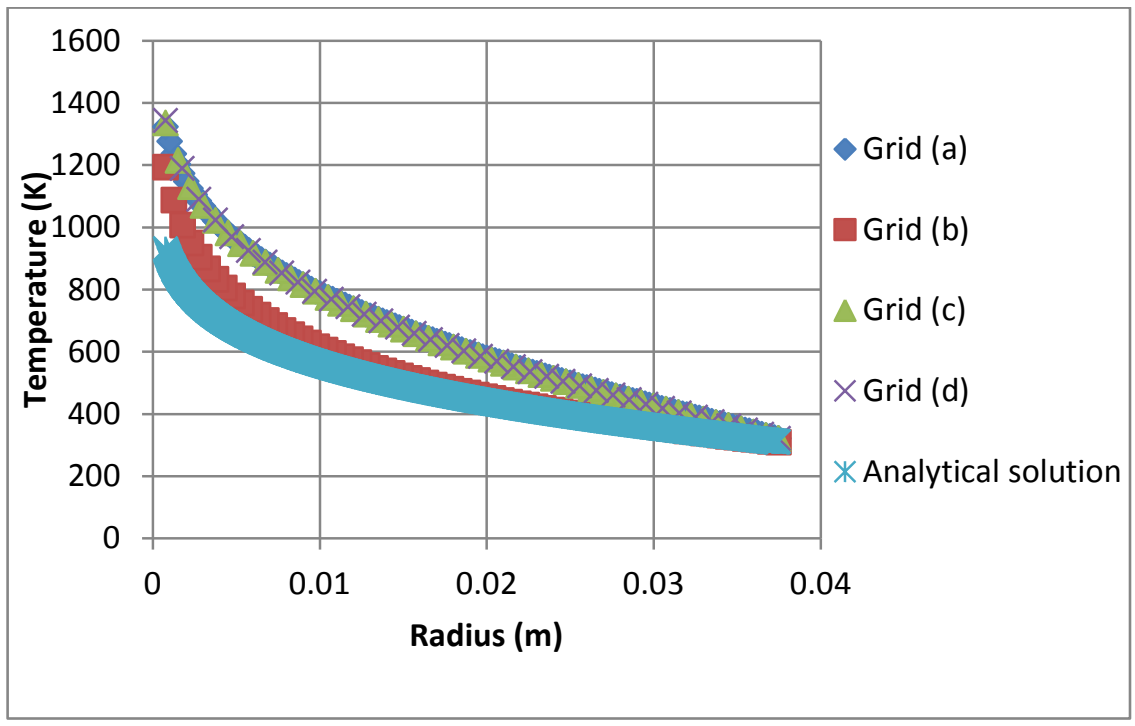

Fig.3: Mesh type effect

The slip flow temperature profiles for different meshes a, b, c and d areshown in Figure 3.The temperature distribution along the radius is calculated, by the Fluent code and compared with the analytical solution (section 3 ). It is found that the results of the mesh (b) or structured with a progressive refinement is more precise than meshes $\mathrm{a}, \mathrm{c}$ and $\mathrm{d}$.

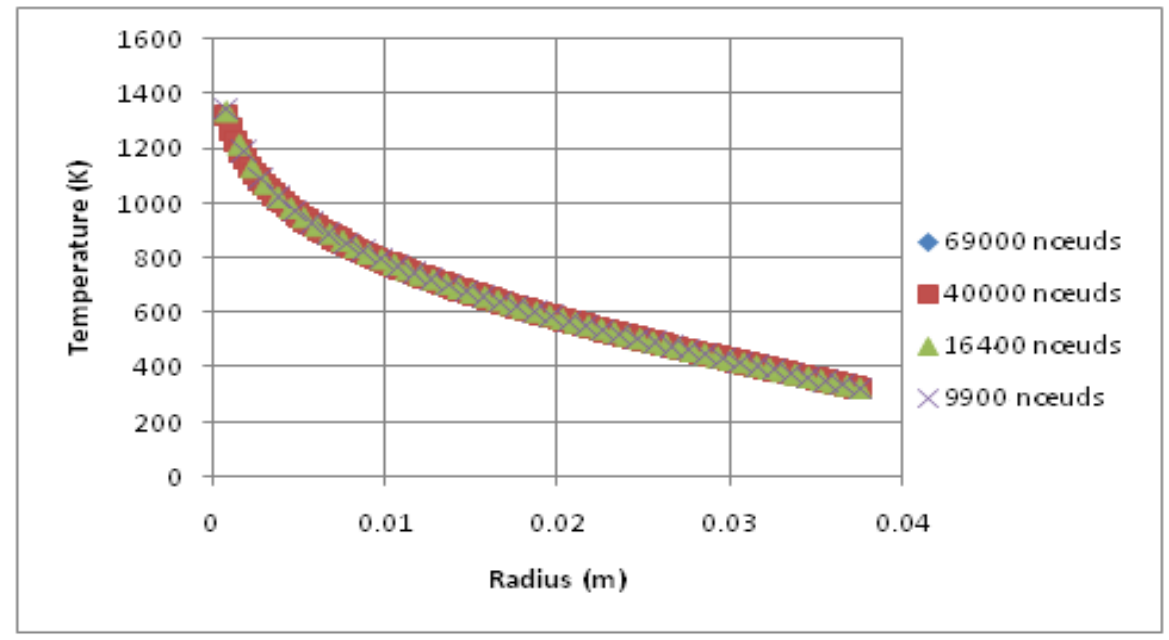

Fig.4:Effect of mesh cells number

After examining the mesh type, the Effect of mesh cells number on the solution is also tested.

Four grids are considered (Figure 4) ranging from 9900 to 69000 nodes. It is seen that the four grids illustratedanalogous behavior, but from the number of 15800 nodes it can be noted that the continuity of the points in the vicinity of the wire in the temperature distribution is not ensured (the points are more spaced), which risks losing significant information in this wire region and consequently the knudsen layer. The grids with 69000 nodes and 40000 nodes give comparable results and therefore the last mesh is choosed throughout this work. The problem case described above is then submitted for computation and a converged solution is reached after performing about 800 iterations. 


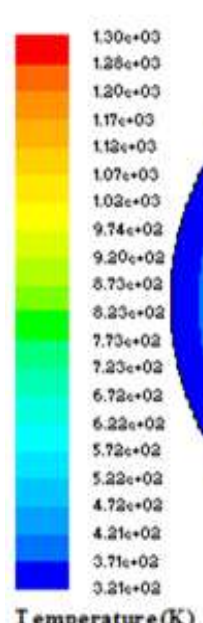

T emperature $(K)$

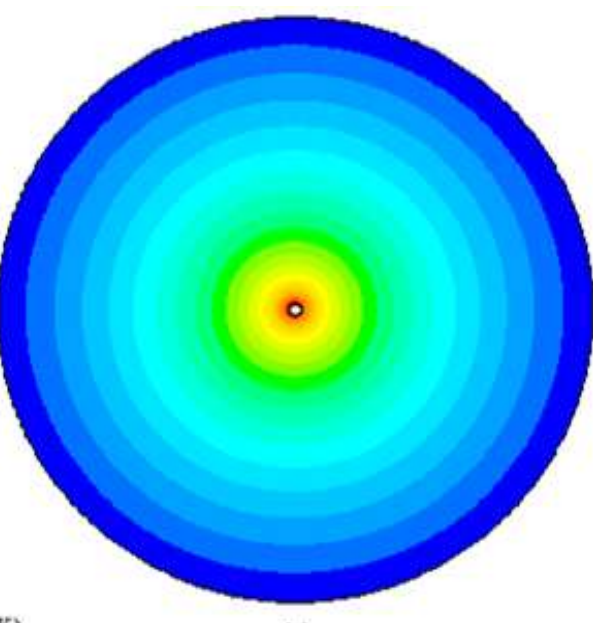

(a)

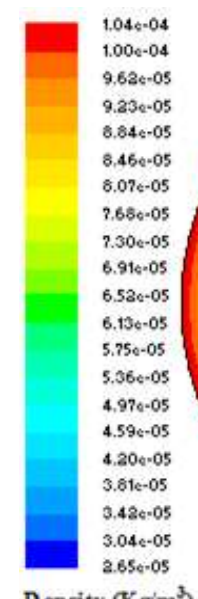

Density $\left(\mathrm{K}_{\mathrm{g}} \mathrm{m}^{3}\right)$

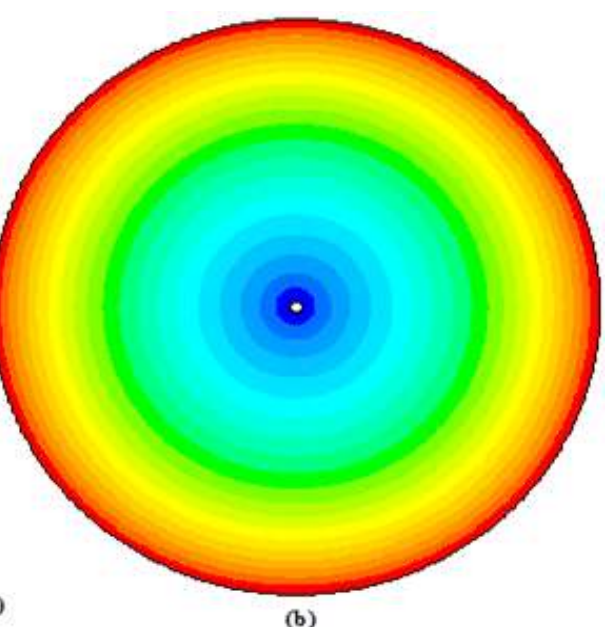

(b)

Fig.5: Temperature (a) and Density (b) Contours
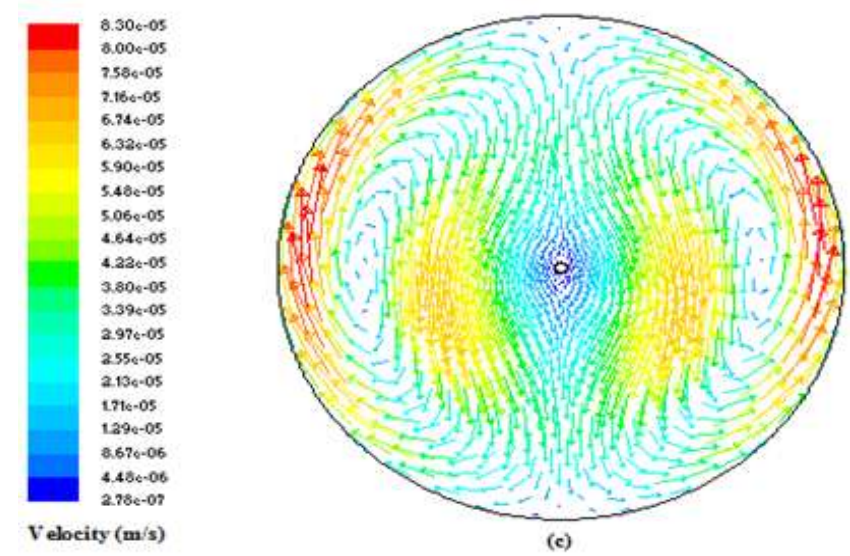

Fig.6:Velocity vectors

Fig. 5 and Fig.6 shows the contours of temperature and density and the velocity vectors of the filament temperature of $2700 \mathrm{~K}$, it can be observed that the temperature (Figure 5(a)) at its maximum in the vicinity of the filament and gradually decrease to reach its minimum at the outside cylinder. The density distribution (Figure $5(b))$ is opposite of that of the temperature, the heating of the internal cylinder causes a decrease of density in the nearness of the inner hot cylinder which engenders a local variation in the density and therefore in the gas mass from the internal to the external wall. It can be seen in the same figure that the density augments monotonically from the inner hot wall to the outer cold wall. This is in good agreement with the temperature results.

The velocity vectors (Figure 6) show that even thought the magnitude of the velocity is low inside the cell; its maximum is attained at the outside cylinder $\left(8.30 .10^{-5}\right)$; it illustrates that there is a macroscopic movement of the gas that was produced, from the internal to the external cylinder.

Fig.7 shows the temperature distributions in terms of radius obtained by using the Navier-Stokes-Fourier calculation (Fluent computer code) and the analytical solutionsubjected to jump boundary conditions. It can be seen that the qualitative behavior of the temperature distribution, given by the two methods, is very similar. But the results show a discrepancy in the vicinity of the filament. The temperature distribution obtained by the code Fluent shows more precision than the analytical solution in particular close to the hot cylinder (near the Knudsen layer). 


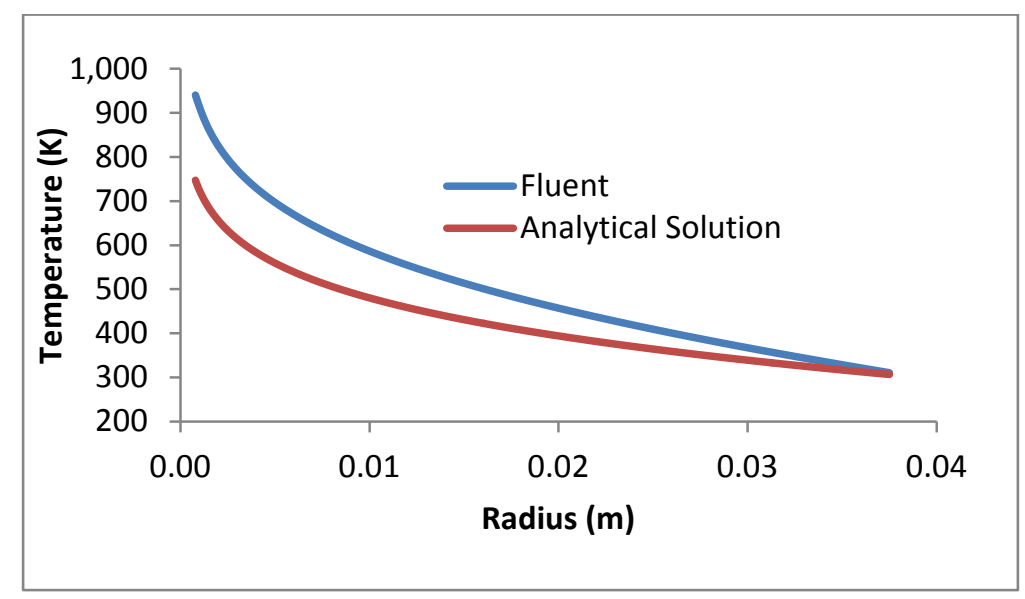

Fig.7: Temperatures distributions obtained by fluent code and the analytical solution.

\section{Conclusion}

The simulation of slip flow through a rarefied nitrogen gas confined between coaxial cylinders is presented on the basis of the Navier-stokes-Fourier equations by using the CFD computer code Fluent.

The influence of the mesh type and the number of nodes on the solution has been examined. It is found that the mesh structured with a progressive refinement is more accurate.

The behavior of the temperature, density, and velocity are analyzed. The results show a discrepancy in the vicinity of the filament, but the qualitative behavior is similar for both methods. The temperature distribution obtained by the computer code Fluent shows more precision than the analytical solution in particular close to the hot cylinder (near the Knudsen layer).

\section{References}

[1] M, Pozarnik and L. S kerget, "Simulation of gas-solid particle flows by boundary domain integral method", Engineering Analysis with Boundary Elements, pp. 939-949, 2002.

[2] P. J, Sun, J.Y. Wu, P. Zhang, L. Xu and M.L. Jiang, "Experimental study of the influences of degraded vacuum on multilayer insulation blankets", Cryogenics 49, pp. 719-726, 2009. https://doi.org/10.1016/j.cryogenics.2009.09.003

[3] G. E. Karniadakis and A. Beskok, "Microflows: fundamentals and simulation", Springer-Verlag, New York, 2002.

[4] J. Pitakarnnop, S. Geoffroy, S. Colin, L. Baldas, "Slip flow in triangular and trapezoidal microchannels", Int .J. Heat Technol, Vol. 26, pp. 167-174, 2008.

[5] V.Leontidis, J.Chen, Baldas, S.Colin, "Numerical design of a Knudsen pump with curved channels operating in the slip flow regime", Heat Mass Transfer, 2014. https://doi.org/10.1007/s00231-014-1314-4

[6] Fluent. Inc., Fluent documentation, www.fluent.com.

[7] S.V. Patankar, "Numerical Heat Transfer and Fluid Flow”, McGraw-Hill, 1980.

[8] S. Boutebba, W. Kaabar and R. Hadjadj, "Fluid Flow Modeling in a Gas-Filled Optical Cell”, Chem. Bull. POLITEHNICA Univ Timisoara, Vol. 56, pp. 71-74, 2011.

[9] S Boutebba, W Kaabar, "Conductive Heat Transfer through a Rarefied Gas Confined Between Two Coaxial Cylinders", in Proc. 2015 International Conference on ChemicalCivil and Environmental Engineering, 2015, pp. 7982.

[10] I.Graur, MT.Ho, and M.Wuest, "Simulation of the transient heat transfer between two coaxial cylinders", J. Vac. Sci. Technol. A 31, 061603, 2013. https://doi.org/10.1116/1.4818870

[11] Mieussens, "L. Discrete-velocity models and numerical schemes for the Boltzmann-BGK equation in plane and axisymmetric geometries", J Comput Phys. 162(2), pp. 429-66, 2000. https://doi.org/10.1006/jcph.2000.6548 